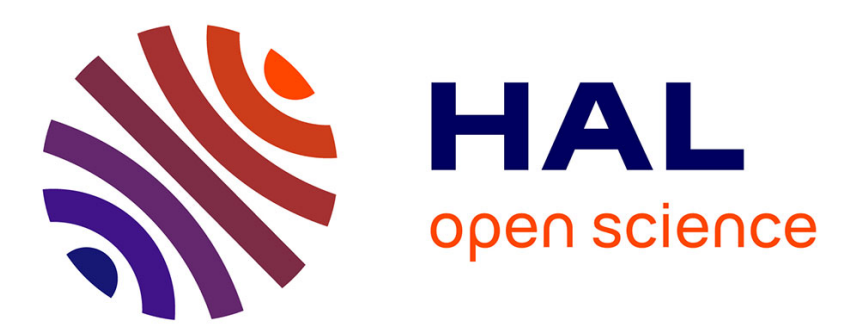

\title{
Interactions mécaniques et physico-chimiques aux interfaces de liaisons céramo-métalliques en fonction de la technique d'assemblage
}

\author{
G. Lascar
}

\section{- To cite this version:}

G. Lascar. Interactions mécaniques et physico-chimiques aux interfaces de liaisons céramo-métalliques en fonction de la technique d'assemblage. Journal de Physique IV Proceedings, 1993, 03 (C7), pp.C71077-C7-1086. 10.1051/jp4:19937169 . jpa-00251798

\section{HAL Id: jpa-00251798 https://hal.science/jpa-00251798}

Submitted on 1 Jan 1993

HAL is a multi-disciplinary open access archive for the deposit and dissemination of scientific research documents, whether they are published or not. The documents may come from teaching and research institutions in France or abroad, or from public or private research centers.
L'archive ouverte pluridisciplinaire $\mathbf{H A L}$, est destinée au dépôt et à la diffusion de documents scientifiques de niveau recherche, publiés ou non, émanant des établissements d'enseignement et de recherche français ou étrangers, des laboratoires publics ou privés. 


\title{
Interactions mécaniques et physico-chimiques aux interfaces de liaisons céramo-métalliques en fonction de la technique d'assemblage
}

\author{
G. LASCAR
}

ISGMP, Laboratoire LM2P, Université de Metz, Ile du Saulcy, 57045 Metz cedex, France

\begin{abstract}
:
The joining between ceramics and metallic alloys can be analysed by three fundamentals aspects.

- The thermodynamic aspects of physical chemistry and mechanic.

- The kinetic aspects of the physical chemistry reaction.

- The electrics aspects.

On the outside specifics reactions physical chemistry and mechanical of junctions developped between ceramics and metallic alloys, the reactions is still in existence a function of the joining technical appliqued.

It is distingued :

- The method of junction by wettability of liquide metallic interlayer and junction by technical a solid state bonding.

- The reactives and non reactives junctions.

The work set out underline the different macromechanic, reactionnal aspects that can explain the adhesion between certain ceramics and metallic alloys. We study the influence of residual stresses, of the nature of components in the area of ceramic/metal interlayer on the lile duration of junction.
\end{abstract}

\section{I / INTRODUCTION.}

Deux techniques de liaison sont mises en oeuvre. Le brasage réactif direct sous vide (liaison à l'état liquide réactive, ê la thermocompression (liaison à l'état solide réaclive ou non réactive). Ces méthodes permettent de réaliser des assemblages entre des céramiques de type oxydes ou non oxydes, verre, vitrocéramiques et des alliages métalliques (ferreux, cuivreux, alliages d'aluminium, titane, zirconium) $/ 1-2 /$. Les verres et vitrocéramiques sont assemblés aux substrats métalliques uniquement par thermocompression. $/ 3-4 /$. Les matériaux de base (alliages métalliques, céramiques) sont choisis en fonction de la différence de propriétés élastiques (E), coefficient de dilatation moyen linéaire qui existe entre eux afin d'analyser l'influence des aspects macromécaniques sur les critères de rupture des liaisons. Les métaux d'apport sont définis a partir des aspects thermodynamiques (liaison réactive ou non réactive). 
Les différents couples analysés sont:

Pour la mise en ocuvre de la méthode de liaison à l'ćtat liquide (liaison réactive).

- $\beta \mathrm{Si}_{3} \mathrm{~N}_{4}, \beta \mathrm{SiC}$ oxydé ou non, $\alpha \mathrm{SiC}, \mathrm{ZrO}_{2}+\mathrm{Al}_{2} \mathrm{O}_{3} /$ aciers, cupro - aluminium, titane et alliages, zirconium et alliages.

Pour les assemblages réalisés selon la technique de thermocompression

(liaison à l'état solide, liaison réactive ou non réactive).

- Les mêmes matériaux de base + verres et vitrocéramique / aciers ou alliages ferro - nickel.

Les aspects réactionnels qualitatifs ou semi quantitatifs sont évalués par analyse par diffraction $\mathrm{X}$ selon la méthode des couches et par microanalyse à énergie dispersive. Les interactions macromécaniques sont analysées par mesure du facteur d'intensité critique de contrainte $\left(\mathrm{K}_{\mathrm{IC}}\right)$ de la céramique el de son évolution en fonction de la distance par rapport à l'interface de liaison de la céramique. Ces investigations sont réalisées par indentation VICKERS ce qui permet d'accéder, par calcul, au niveau des contraintes résiduclles locales macroscopiques.

\section{II / PROCEDURE EXPERIMENTALE.}

II - 1 / Asscmblage à l'état liquide.

Un métal d'apport est interposé enire les deux surfaces à assembler. Il présente une température de fusion inférieure à la température de fusion des materiaux de base. La température d'assemblage est donnće par une règle empirique $T_{\mathbf{A}}=$ $\mathrm{T}_{\mathrm{F}}+50 \grave{\mathrm{a}} 100^{\circ} \mathrm{C}$ ( $\mathrm{T}_{\mathrm{F}}$ : température de fusion du métal d'apport).

Les mécanismes fondamentaux initiaux entre le métal d'apport liquide et les surfaces de liaison sont:

- Adsorption physique.

- Adsorption chimique.

- Mouillage et étalement du métal d'apport liquide (goutte sessile)

sur les surfaces solides.

Ces réactions sont essentielles pour que les autres natures de réactions, pouvant être prévues thermodynamiquement d'après les diagrammes d'équilibre de phases, puissent se produire.

De ce point de vue, les réactions qui se développent à l'interface métal de base / métal d'apport liquide doivent être distinguées.

En effel, la nature des liaisons chimiques assurant la cohésion des céramiques et des alliages métalliques est différente. De ce fait, les mécanismes élémentaires fondamentaux de surface entre la phase liquide et les deux interfaces de liaison seront différents.

L'adhésion initiale du métal d'apport liquicle sur les surfaces solides est définie par l'angle de contact $\left(\theta_{\mathrm{c}}\right)$ généré par l'équilibre de trois énergies superficielles $\left(\gamma_{\mathrm{L} / \mathrm{V}}\right.$ : énergie de surface liquide / vapeur, $\gamma_{\mathrm{S} / \mathrm{L}}$ : énergie de surface solide / liquide, $\gamma_{\mathrm{S}} / \mathrm{V}$ : énergie de surface solide / vapeur) mises en évidence par la goutle SESSILE (figure 1). En première approximation, l'angle de contact s'exprime : 
Pour les alliages métalliques :

Pour les céramiques :

$$
\cos \theta_{\mathrm{c}}=\frac{\gamma_{\mathrm{S} / \mathrm{V}}-\gamma_{\mathrm{S} / \mathrm{L}}}{\gamma_{\mathrm{L} / \mathrm{V}}} ; \mathrm{Wad}=\gamma_{\mathrm{N}}\left(1+\cos \theta_{\mathrm{c}}\right)
$$

$$
\cos \theta_{\mathrm{c}}=\frac{\gamma_{\mathrm{c}}-\gamma_{\mathrm{i}}}{\gamma_{\mathrm{m}}}: \mathrm{Wad}=\gamma_{\mathrm{m}}\left(1+\cos \theta_{\mathrm{c}}\right)
$$

Pour qu'une interface (i) puisse se former, il faut vérifier que :

$$
\gamma_{\mathrm{i}}<\gamma_{\mathrm{c}}+\gamma_{\mathrm{m}} ; \quad \mathrm{Wad}=\gamma_{\mathrm{c}}+\gamma_{\mathrm{m}}-\gamma_{\mathrm{i}}
$$

Donc le mouillage de la phase liquide sur les surfaces solides, soit l'adhésion, évolue en fonction de l'angle de contact, défini par les énergies superficielles.

$\theta=0^{\circ} ; \mathrm{Wad}=2 \gamma_{\mathrm{N}} \mathrm{V}$ ou $2 \gamma_{\mathrm{m}} ; \gamma_{\mathrm{c}}-\gamma_{\mathrm{m}}=\gamma_{\mathrm{i}}$ soit $\gamma_{\mathrm{m}}-\gamma_{\mathrm{c}}+\gamma_{\mathrm{i}}=0$

$\theta=90^{\circ} ; \mathrm{Wad}=\gamma_{\mathrm{L}} / \mathrm{N}$ ou $\gamma_{\mathrm{m}} ; \gamma_{\mathrm{c}}=\gamma_{\mathrm{i}}$

$\theta=180^{\circ} ; \mathrm{Wad}=0 ; \gamma_{\mathrm{m}}+\gamma_{\mathrm{c}}=\gamma_{\mathrm{i}}$ soit $\gamma_{\mathrm{m}}+\gamma_{\mathrm{c}}-\gamma_{\mathrm{i}}=0$

La création de l'interface métal liquide / céramique nécessite un arrangement de la distribution des charges électrostatiques surfaciques de la céramique afin de permettre la formation de liaison du type de VAN DER WALIS (adsorption physique). Toutefois, la valcur de langle de contact $\left(\theta_{\mathrm{C}}\right)$ est très élevée entre les métaux liquides et les céramiques. Il est nécessaire de diminuer $\left(\gamma_{\mathrm{c}}\right)$ afin de diminuer $\left(\theta_{c}\right)$ et $\gamma_{i}$. Ceci explique que les mélaux d'apport utilisés sont choisis en fonction de la réactivité, avec la céramique, d'éléments d'addition contenus dans ceux - ci et de leur compatibilité métallurgique avec le métal de base. Les éléments thermodynamiquement réactifs avec les céramiques de type oxydes ou non oxydes souvent utilisés sont le titane et le zirconium, d'ou les réactions :

$$
\begin{aligned}
& \mathrm{Si}_{3} \mathrm{~N}_{4}+4 \mathrm{Ti} \longrightarrow 4 \mathrm{TiN}+3 \mathrm{Si} \\
& \mathrm{SiC}+\mathrm{Ti} \stackrel{-}{\rightarrow} \mathrm{TiC}+\mathrm{Si} \\
& \mathrm{ZrO}_{2}+2 \mathrm{Ti} \stackrel{-\rightarrow}{\rightarrow} 2 \mathrm{TiO}+\mathrm{Zr} \\
& \mathrm{Al}_{2} \mathrm{O}_{3}+3 \mathrm{Ti} \stackrel{\rightarrow}{\rightarrow} 3 \mathrm{TiO}+2 \mathrm{Al} \\
& \Delta G_{R}^{\circ}=4 \Delta G^{\circ}(\mathrm{TiN})-\Delta \mathrm{G}^{\circ}\left(\mathrm{Si}_{3} \mathrm{~N}_{4}\right) \\
& \Delta \mathrm{G}_{\mathrm{R}}^{\circ}=\Delta \mathrm{G}^{\circ}(\mathrm{TiC})-\Delta \mathrm{G}^{\circ}(\mathrm{SiC}) \\
& \Delta \mathrm{G}_{\mathrm{R}}^{\circ}=2 \Delta \mathrm{G}^{\circ}(\mathrm{TiO})-\Delta \mathrm{G}^{\circ}\left(\mathrm{ZrO}_{2}\right) \\
& \Delta G_{R}^{\circ}=3 \Delta G^{\circ}(\mathrm{TiO})-\Delta G^{\circ}\left(\mathrm{Al}_{2} \mathrm{O}_{3}\right) \\
& \Delta G_{R}^{\circ}=R T L_{n} \frac{[a A l]^{2}[a T i O]^{3}}{\left[a A_{2} \mathrm{O}_{3}\right][a T i]^{3}}=R T I . n=\frac{[a A l]^{2}}{[a T i]^{3}} \\
& \Delta \mathrm{G}_{\mathrm{R}}^{\circ}=\mathrm{RTL}_{\mathrm{n}} \frac{[\mathrm{aZr}][\mathrm{a} \mathrm{TiO}]^{2}}{[\mathrm{aTi}]^{2}[\mathrm{a} \mathrm{ZrO} 2]}=\operatorname{RTLn}=\frac{[\mathrm{a} \mathrm{Zr}]^{2}}{[\mathrm{aTi}]^{2}} \\
& \Delta G_{R}^{\circ}=R T L_{0} \frac{[a S i][a T i C]}{[a T i][a S i C]}=R T L_{n}=\frac{[a S i]}{[a T i]}
\end{aligned}
$$




$$
\Delta G_{R}^{\circ}=R T L_{n} \frac{[a S i]^{3}\left[a \operatorname{TiN}^{4}\right.}{\left[a T_{i}^{4}\left[\mathrm{aSi}_{3} \mathrm{~N}_{4}\right]\right.}=R T \operatorname{Ln}=\frac{[\mathrm{a} \mathrm{Si}]^{3}}{[\mathrm{aTi}]^{4}}
$$

La solubilité de l'oxygène, dans le titane ou le zirconium revêt une grande importance sur la réaction et la nature de l'oxyde formé lors de la réaction du titane avec les céramiques de type oxydes. Ceci explique que pour la réalisation des liaisons réactives directes sous vide à l'état liquide les éléments réactifs essentiels, le titane ou le zirconium, sont inclus dans des alliages du type Ag $\mathrm{Cu}, \mathrm{Cu}-\mathrm{Al}$ - Si, Ag - Cu - Sn. Le cuivre forme des composés du type siliciure de cuivre avec $\mathrm{Si}_{3} \mathrm{~N}_{4}$ et $\mathrm{SiC}$ ou $\mathrm{CuAlO}_{2}$ avec $\mathrm{Al}_{2} \mathrm{O}_{3}$. Ũn dépót de titane (C. $\mathrm{V}$. D., évaporation, P.V.D.) peut être réalisé sur la surface de la céramique $15 /$. $D^{\prime}$ autres réactions du type évaporation - condensation, échanges, addition, échanges électroniques peuvent se produire.

Les investigations sont réalisées pour des éprouvettes assemblées par liaison à l'êtat liquide en utilisant des alliages $\mathrm{Ag}-\mathrm{Cu}-\mathrm{Ti}$ contenant soit $1,7 \%$ ou $4,5 \%$ de titane.

\section{II - 2 / Assemblage à l'étal solide (thermocompression).}

La liaison est réalisée en interposant un métal d'apport entre les deux surfaces à assembler et en déformant plastiquement celui - ci à une température de $0,5-0,9$ $T_{F}\left(T_{F}\right.$ : température de fusion du mélal d'apport en ${ }^{\circ} \mathrm{C}$ ) $/ 6-7 /$. Les liaisons peuvent être réactives ou non réactives en fonction des métaux d'apports utilisés et des aspects réactionnels thermodynamiques liés au couple ceramique / métal d'apport. Les principaux paramètres de liaisons sont, la lempérature, la contrainte, la vitesse de délormation plastique du métal d'apport, les conditions d'atmosphère, le palier thermique à $T_{D}$ sous $\sigma_{D}$, la nature physico-chimique de la surface de liaison. Les mécanismes d'adhésion du métal d'apport sont de deux natures, mécanique (contrainte de cisaillement générée par le frottement des matériaux), réactive (diffusion, addition, échange, liaison sur des lacunes, chaines pendantes, défauts chargés, défauts de structure de surface, échanges électroniques). En fonclion des conditions technologiques de liaison, des phases liquides peuvent se former à l'interface de liaison si les paramètres de liaison correspondent aux conditions thermodynamiques d'existence d'une phase liquide, l'exemple est donné par le diagramme d'équilibre $\mathrm{Cu}$ - Si qui présente un eutectique. De ce fait, lors d'une thermocompression $\mathrm{SiC} / \mathrm{Cu}$ ou $\mathrm{Si}_{3} \mathrm{~N}_{4} / \mathrm{Cu}$ dans les conditions technologiques de liaison l'un des eutectiques prévus thermodynamiquement peut se former (figures 2 - 4). Il se produira une réaction équivalente pour le système $\mathrm{Ni} / \mathrm{SiC}$. Dans le cas du $\mathrm{Si}_{3} \mathrm{~N}_{4}$, la nature des joints de grains el les réactions qui se développent à ce niveau est prépondérante, pour $\mathrm{SiC}$ les ségrégations intergranulaires jouent un rôle très important. Dans le cas de systèmes non réactifs (exemple : couple $\mathrm{Ag} / \mathrm{Si}$ ou $\mathrm{SiC}$ / Ag, figure 3), l'adhésion est purement mécanique, elle se développe du fait des contraintes de cisaillement et de l'accommodation mécanique et électrique de l'interface de liaison.

Toutes les liaisons analysées, d'un point de vue des réactions physico chimiques et macromécaniques développées aux interfaces, sont réalisées avec 
un métal d'apport A9 ou A5 ou AS1 (alliage aluminium - silicium). L'analyse des couples $\mathrm{Si} / \mathrm{Ag}$ ou Si / Cu est qualitative.

\section{III / RESULTATS.}

Les investigations réalisées (examens métallographiques, indentation VICKERS pour tous les couples, photoélasticimétrie par transparence pour le couple vitrocéramique / $\mathrm{Al} / \mathrm{Fe}-\mathrm{Ni}-\mathrm{Co}$, microanalyse par microsonde à énergie dispersive pour les assemblages brasés, diffraction $X \theta-2 \theta$ ) montrent :

Pour les assemblages brasés (liaisons réactives) :

- Une dissolution importante des métaux de base en alliage de titane et de zirconium dans le métal d'apport, elle est moins importante pour l'acier (figure 5).

- Une ségrégation très importante de titane à l'interface des céramiques, $\mathrm{Si}_{3} \mathrm{~N}_{4}$ ou $\mathrm{ZrO}_{2}$, la profondeur de la zone de réaction métal d'apport / céramique est de l'ordre de 3 à $6 \mu \mathrm{m}$.

- La formation de composés $\mathrm{Cu}$ - Ti dans la zone de dissolution métal de base en alliage de titane ou de zirconium.

- Une diminution importante du facteur d'intensité critique de contrainte de la céramique en fonction de la distance par rapport à l'interface de liaison de la céramique pour tous les couples et un niveau de contraintes résiduelles important (figures 10 - 11).

Pour les assemblages réalisés par thermocompression (Iigure 6) :

- La zone de réaction présente une profondeur qui évolue en fonction de la nature des matériaux en présence (figures $7-8$ ), des paramètres de liaison, de la nature des réactions et de la cinétique de celle - ci. L'exemple peut être donné pour le couple acier / $\mathrm{AS} 1 / \mathrm{SiC}$, pour lequel la zone de réaction à l'interface de l'aluminium / SiC est de l'ordre de $5000 \AA$ environ, et de quelques microns à l'interface de l'acier de nuance $Z 30 C 13$ ou autre nuance d'acier, d'alliages ferreux, pour un palier de 30 minutes a $550^{\circ} \mathrm{C}$ et une contrainte de déformation plastique du métal d'apport de l'ordre de 30 à $50 \mathrm{MPa}$, alors qu'une liaison très réactive du type nickel / $\mathrm{SiC}$ provoque la fissuration in situ de la cerramique (formation des siliciures de nickel et de l'eutectique $\alpha+\gamma$ ou autres, des composés définis). Le cuivre forme aussi un eutectique $\mathrm{Cu} /$ siliciures de cuivre lors des liaisons réactives formées avec $\mathrm{SiC}$, toutefois la cinétique de réaction est inférieure à celle observée pour le couple $\mathrm{Ni} / \mathrm{SiC}$. Malgré la présence d'eutectique et de composés définis, la liaison présente une bonne adhésion, sans fissuration in situ. La réaction du métal d'apport avec le métal de base acier inoxydable ou base nickel peut être à l'origine de la présence de nickel au niveau de l'interface de liaison de SiC. Dans ce cas, il est observé des eutectiques, prévus par le diagramme $\mathrm{Ni} / \mathrm{Si}$, dans la zone de réaction $\mathrm{SiC} /$ mélal d'apport et une fissuration du $\mathrm{SiC}$ (figure 9).

- Il existe une diminution du facteur d'intensité critique de contrainte $\left(\mathrm{K}_{\mathrm{IC}}\right)$ de la céramique (figure 12) près de la zone de réaction, quelle que soit la nature du couple céramo - métallique. Toutefois, cette variation de $\mathrm{K}_{\mathrm{IC}}$ est en général moins importante par rapport à l'évolution du KIC déterminée pour les assemblages réalisés à l'état liquuide.

Les calculs du niveau de contraintes résiduelles réalisés à partir des mesures d'indentation définissent des valeurs de contraintes résiduelles qui varient en 
fonction de la nature des couples céramo - métalliques. Celles - ci peuvent ètre du même ordre de grandeur pour un couple spêcifique par rapport au niveau des contraintes résiduelles déterminés pour les assemblages réalisés à l'état liquide.

\section{IV/CONCLUSIONS.}

Les travaux présentés montrent que les liaisons réactives réalisées à l'état liquide affectent la céramique (diminution du $\mathrm{K}_{\mathrm{IC}}$ dans la zone de liaison céramique / métal d'apport, fissuration de la céramique possible du fait des contraintes résiduelles et du comportement thermomécanique des composés formés qui sont en général fragiles). Les liaisons réactives effectuées par thermocompression fragilisent moins les céramiques, en général, sauf si il se forme une phase liquide selon une cinétique importante (cas du couple $\mathrm{SiC} / \mathrm{Ni}$ ).

Pour les couples analysés $\mathrm{Ti} / \mathrm{S} \mathrm{i}_{3} \mathrm{~N}_{4}, \mathrm{Zr} / \mathrm{Si}_{3} \mathrm{~N}_{4} ;$ il est observé une dissolution importante du titane dans le métal d'apport $\mathrm{Ag}-\mathrm{Cu}-\mathrm{TiC}$ et une ségrégation importante du titane à l'interface de liaison $\mathrm{Si}_{3} \mathrm{~N}_{4} /$ métal d'apport.

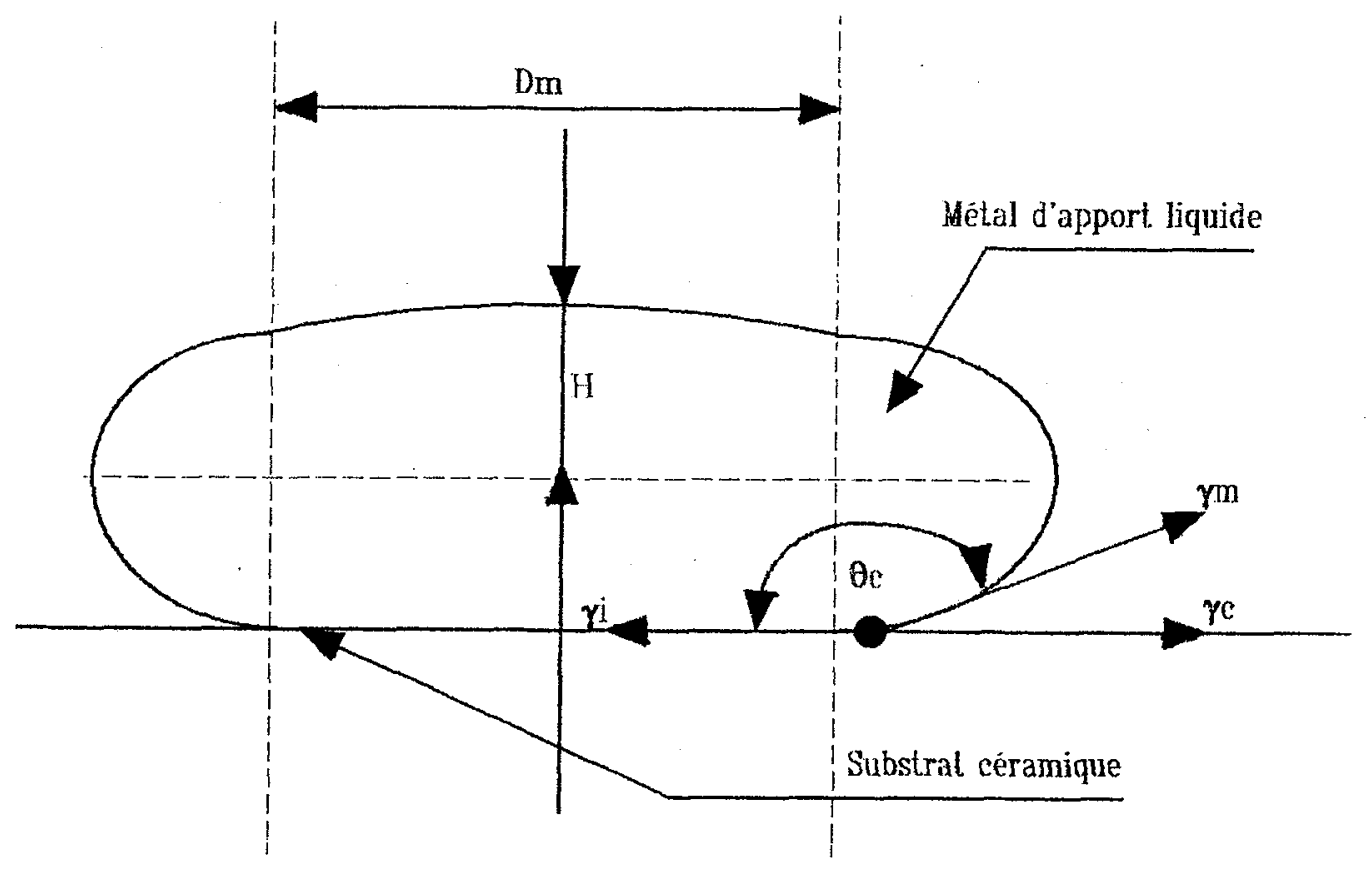

FIGURE 1 - SCHEMA DE LA GOUTTE SESSILE. 


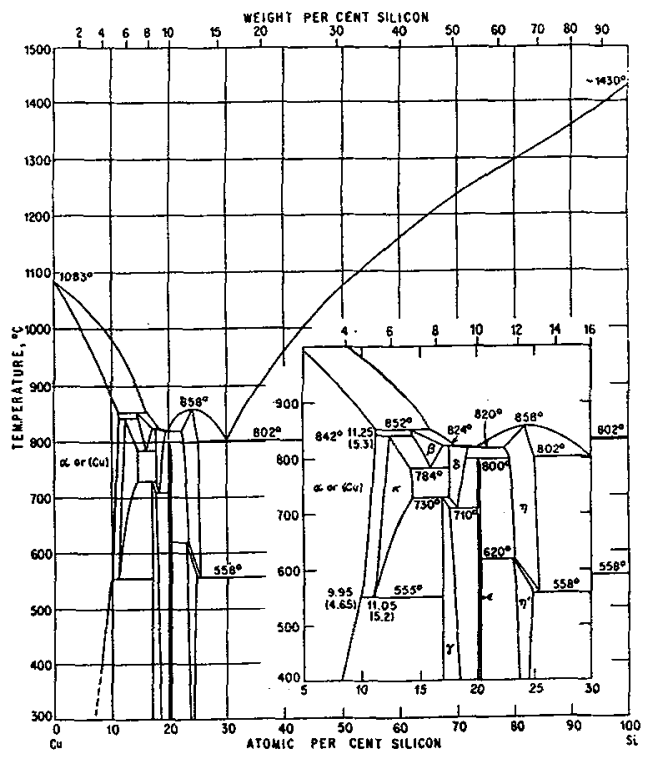

FIGURE 2 - DIAGRAMME D'EQUILIBRE DE PHASES BINAIRES Si-CU.

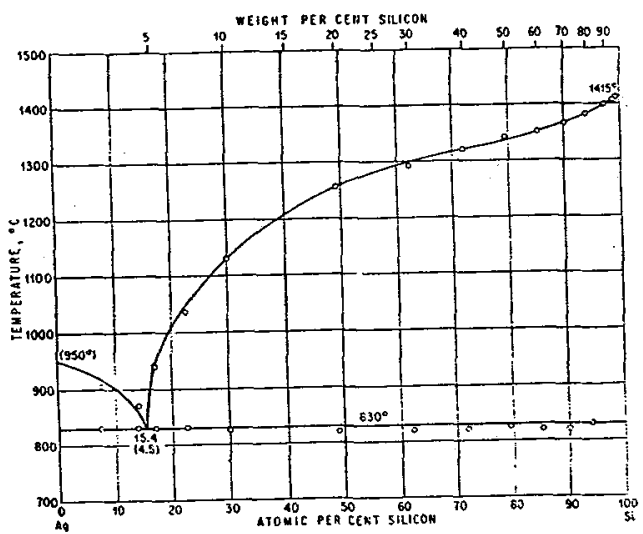

FIGURE 3 - DIAGRAIME D'EQUILIBRE DE PHASE BIAAIRE Ag - Si. 


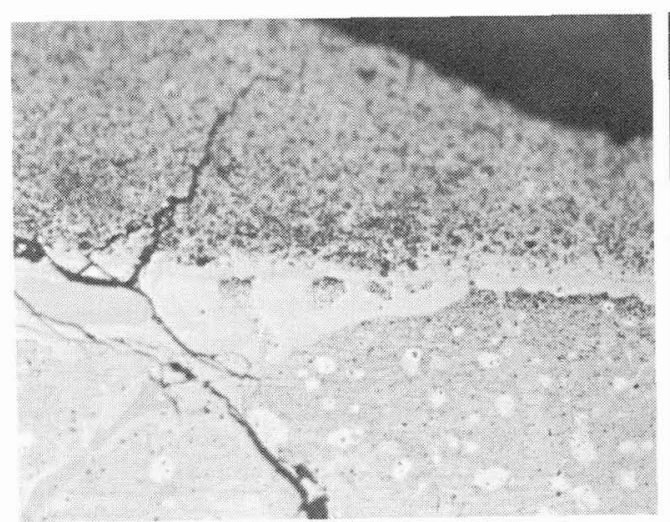

$\mathrm{x} 500$

FIGURE 4-MICROPHOTOGRAPHIE A L'INTERFACE SiC/CU.

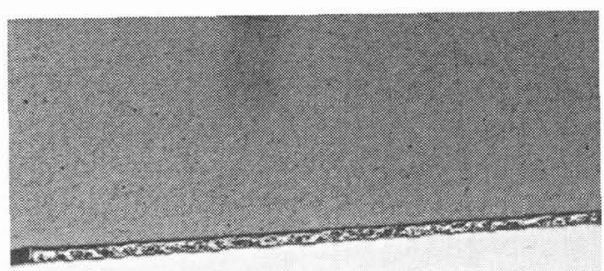

$\mathrm{x} 11$

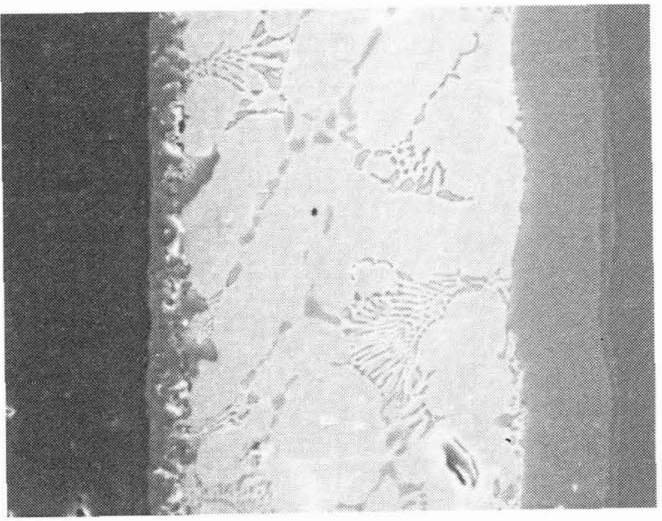

$x 800$
FIGURE 5 - COUPLE TITANE

/ Ag- $\mathrm{Cu}-\mathrm{Ti} /$ nitrure de silicium.

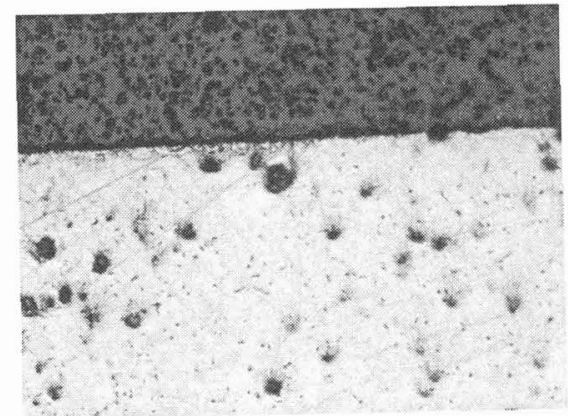

$x 200$

\section{FIGURE 6 -}

FIGURE 7 - SiC / AS1

ASSEMBLAGE ACIER Z30C13 / SIC REALISE PAR THERMOCOMPRESSION.

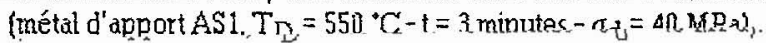

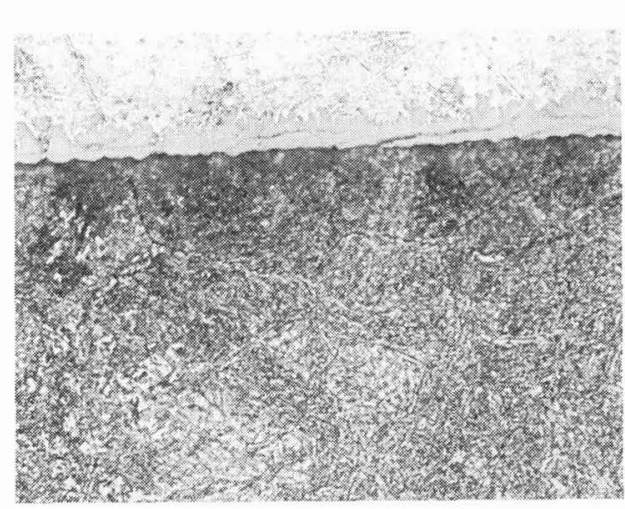

$\times 500$

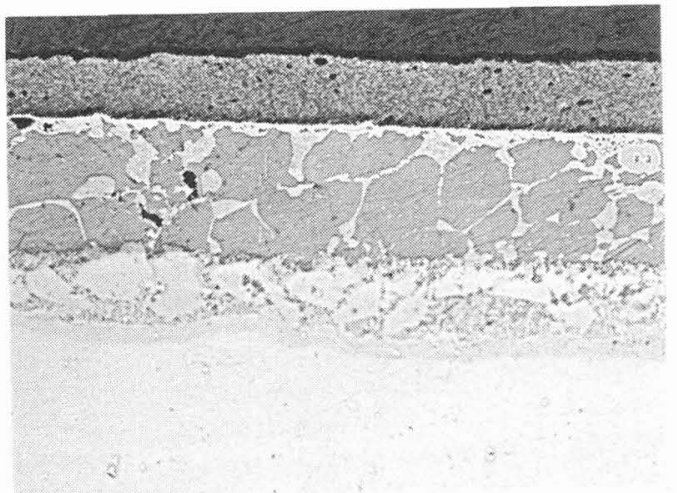

$x 200$
FIGURE 8 - COUPLE Z30C13/Al-Si REALISE PAR. THERMOCOMPRESSION.
FIGURE 9 - COUPLE BASE Ni / Cu / SiC REALISE PAR THERMOCOMPRESSION. 


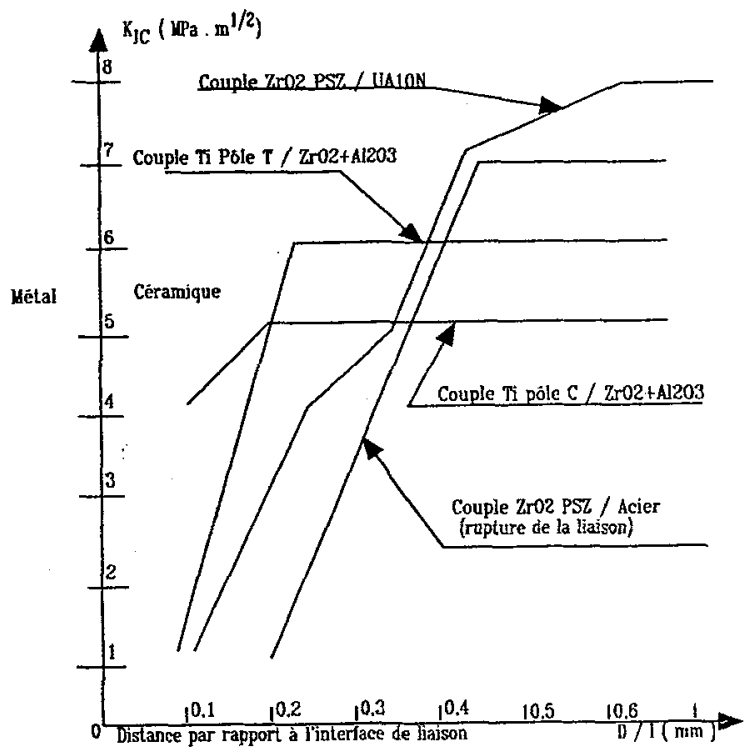

FIGURE 10 - EVOLUTION DU K $\mathrm{K}_{\mathrm{IC}}$ DE LA CERAMIQUE MESUREE

PAR INDENTATION VICKERS EN FONCTION DE LA DISTANCE

PAR RAPPORT A L'INTERFACE DE LIAISON (assemblages réalisés à l'átat liquide).

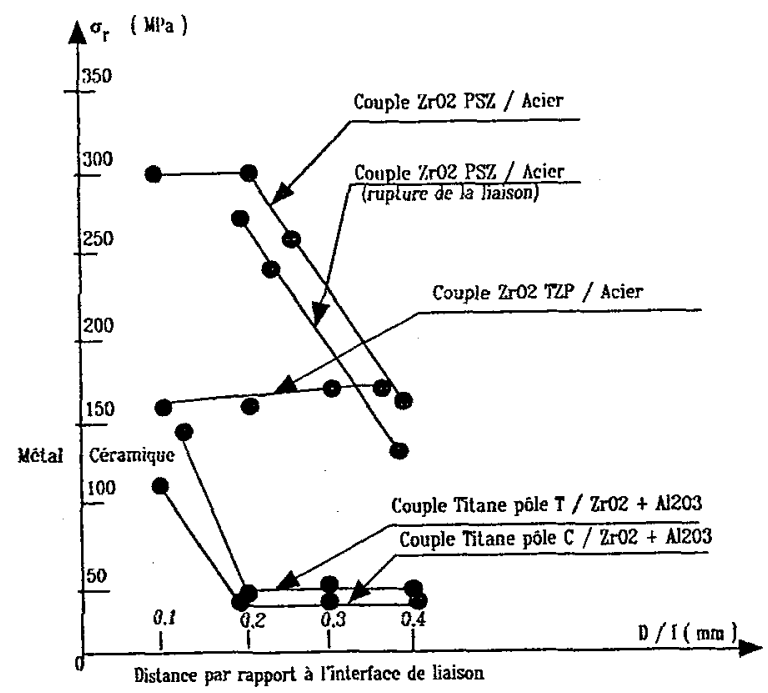

FIGURE 11 - EVOLUTION DU NIVEAU MACROSCOPIQUE DE CONTRAINTES RESIDUELLES EN FONCTION DE LA DISTANCE PAR RAPPORT A LINTERFACE (assemblages à l'état tiquide). 


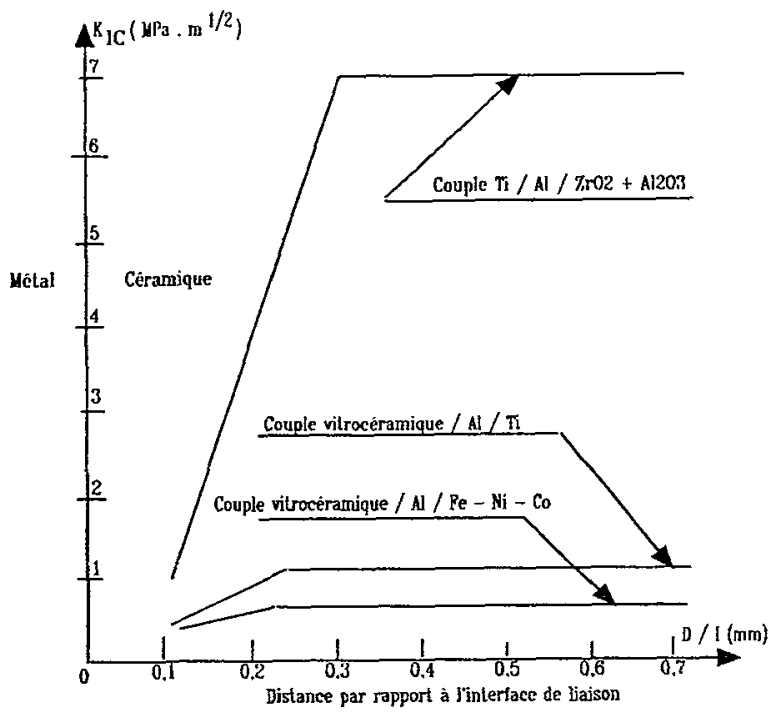

FIGURE 12 - EVOLUTION DU KIC DE LA CERAMIQUE MESUREE

PAR INDENTATION VICKERS EN FONCTION DE LA DISTANCE PAR RAPPORT A L'INTERFACE DE LIAISON (assemblages réalisés à l'état solide).

\section{REFERENCES BIBLIOGRAPHIQUES.}

[1] LASCAR G.. Contribution à l'étude des assemblages entre céramiques et alliages métalliques. Analyse des interactions mécaniques ef physico chimiques. Thèse de Doctoral de l'Université de Metz en Sciences de l'Ingénieur soulenue le 21 décembre 1989.

[2] LASCAR G.. Analysis of reactions in ceramic - melallic joint. Welding Interaational 19926 (3) - p. $194-11$.

[3] LASCAR G.. Etude des liaisons entre métaux et une vitrocéramique. Application à la réalisation d'ensembles électro - optiques. Mémoire et Etudes Scientifiques - Revue de la Métallurgie - juillet/août 1985 - pp. 347-363.

[4] KIOMP J. T.. Chemistry and kinelic aspects of metal-ceramic diffusion bonding. Conference joining Glass and Metal Bad NAUHEIM.

[5] SANTELLA L., PAK J. J.. Brazing titanium - vapor - coated zirconia. Welding Research supplemen - avril 1993 - supplément pp. 165-172.

[6] LASCAR G.. Method for producing a fluidtight and heat curable seal between metal objects and a body of vitroceramic material. Brevet demande U.S. n 07/119253 - 16 mars 1989 - Société QUANTEL.

[7] LASCAR G., BROUSSAUD D., THOREL A., CAIllETAUD G., ROLAND J. J.. Reliability of metal glass - ceramic junctions made by solid state bonding. Journal de physique - Colloque C1 - février 1986 - supplénent n² 2 tome 47 - pp. 139. 